\title{
Colistin-resistant microorganisms and cystic fibrosis: microbiological surveillance in an Italian Children's Hospital
}

\author{
Anna Lisa Montemari, ${ }^{1}$ Vanessa Tuccio Guarna Assanti, ${ }^{1}$ Giulia Linardos, ${ }^{1}$ Giovanni Di Bonaventura, ${ }^{2}$ \\ Gabriella Ricciotti, ${ }^{1}$ Ersilia Vita Fiscarelli ${ }^{1}$ \\ ${ }^{1}$ Cystic Fibrosis Microbiology Laboratory, Department of Laboratories, Bambino Gesù Children's Hospital, IRCCS, \\ Rome; ${ }^{2}$ Department of Medical, Oral and Biotechnological Sciences; Centre of Excellence on Ageing and \\ Translational Medicine, “G. d'Annunzio” University of Chieti-Pescara, Chieti, Italy
}

\begin{abstract}
Summary
Several advances in the medical field are often dependent on the ability to fight infections with the use of antibiotics, including joint replacements, organ transplants, and cancer therapy. The capacity of the bacteria to adapt to and escape from the mechanisms of action of antibiotics makes the antimicrobial resistance a serious public health problem worldwide. Polymyxin E colistin has rarely been used because of its nephrotoxicity and neurotoxicity. More recently, the emergence of multi-drug resistant bacteria as carbapenem-resistant Klebsiella pneumoniae, Acinetobacter
\end{abstract}

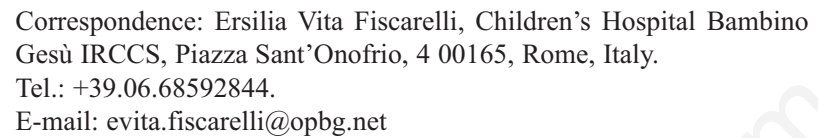

E-mail: evita.fiscarelli@opbg.net

Key words: colistin-resistance, mcr-1, cystic fibrosis.

Acknowledgments: The authors would like to thank Dr. Antonio Battisti and Dr. Alessia Franco from Istituto Zooprofilattico Sperimentale del Lazio e Toscana for supplying the mcr-1 positive E. coli DNA extract, Paula Dorotea Ristagno for technical assistance and Dr. Andreina Santoro for English language editing.

Contributions: ALM, PCR implementation, data analysing, manuscript writing, references search, VTGA, bacteria isolation and identification, susceptibility testing, GL, bacteria isolation and identification, susceptibility testing, GDB, manuscript advising, GR, bacteria isolation and identification, susceptibility testing, EVF, manuscript reviewing.

Conflict of interest: the authors declare no potential conflict of interest.

Funding: none.

Received for publication: 11 March 2019.

Accepted for publication: 14 March 2019.

(C) Copyright A.L. Montemari et al., 2019

Licensee PAGEPress, Italy

Microbiologia Medica 2019; 34:8163

doi:10.4081/mm.2019.8163

This article is distributed under the terms of the Creative Commons Attribution Noncommercial License (by-nc 4.0) which permits any noncommercial use, distribution, and reproduction in any medium, provided the original author(s) and source are credited. baumannii and Pseudomonas aeruginosa and the re-evaluation of its pharmacokinetic properties have led to a resurgence of colistin as a treatment option, contributing to select resistant strains. Investigating the phenomenon of colistin-resistance in gram-negative bacteria, especially $P$. aeruginosa, is now mandatory, particularly after identification of a plasmid-mediated mechanism for the resistance to colistin ( $\mathrm{mcr}$ ) in Enterobacteriaceae strains, a mechanism transferable to other species. In this study, we investigated colistin-resistance in gram-negative bacteria isolated from respiratory secretions of cystic fibrosis patients in follow-up at Children's Hospital Bambino Gesù of Rome.

\section{Introduction}

Colistin, also known as polymyxin E, belongs to the group of antibiotic polypeptides derived from Bacillus polymyxa. Cationic colistin polypeptide interacts with lipopolysaccharide (LPS) of gram-negative bacteria by shifting the calcium and magnesium ions destabilizing the membrane with a permeability increase (18). Commercialized in the fifties, colistin has rarely been used because of its nephrotoxicity and neurotoxicity; however, the emergence of the antibiotic-resistance and the re-evaluation of its pharmacokinetic properties have recently encouraged the reintegration into clinical practice. Colistin is effective in treating infections caused by carbapenem-resistant Escherichia coli and Klebsiella spp. $(5,15)$, and Multi-Drug Resistant (MDR) Acinetobacter baumanni. It is also commonly used for therapy, including aerosol, in patients with cystic fibrosis (CF) and chronic infection by Pseudomonas aeruginosa (20). However, immediately after its re-use, bacteria have developed resistance.

Classical colistin resistance mechanisms include both biochemical and genetic mechanisms. The most frequent is a modification of LPS for the attachment of the 4-amino-4-deoxy-1-arabinose (Lara4N) or the phosphoethanolamine (PEtN) to a phosphate group in the lipid A; this attachment induce an increased net charge leading to a reduction of colistin affinity for polymyxins. The activation of the sensor kinases PhoQ and PmrB under specific stress conditions triggers a phosphorilation of cytoplasmatic regulators PhoP and PmrA which regulate the expression of arnBCADTEF and pmrCAB genes modulating LPS modifications by Lara4N and PEtN. Loss of LPS for mutations in the IpxA, IpxC and $I p x D$ genes, single mutation in the PmrB protein or involvement of OprH, a membrane protein which contributes to the stability of the cellular shell, are other known mechanisms of resistance to colistin, as well as the presence of a capsule, efflux pumps 
expressions and changes in the regulatory loci $p m r A$ and $p h o P$ $(3,28)$. The heteroresistance, namely the resistance to an antimicrobial expressed by a subpopulation in a susceptible population, selects numerous colistin-resistant bacteria.

In 2016, a new mechanism has been discovered in China, where for the first time was described in E. coli a plasmid-mediated mechanism for colistin-resistance, called mcr-1 (22). The $\mathrm{mcr}$ 1 gene encoding for a phosphoethanolamine-transferase is capable of modifying the portion of lipid A of LPS, resulting in the inhibition of the polymyxin's binding to the membrane.

Since the first description, new identifications occurred in many countries, including Malaysia (44), Thailand (12), Vietnam (35), Japan (36), USA (21), as well as in several European countries $(8,13)$. The worldwide spread of $m c r-1$ represents a great concern because the plasmid allows horizontal transmission of the resistance gene and, therefore, a transferable resistance among species. Its presence, in fact, has been detected also in Klebsiella pneumoniae (33), Acinetobacter spp.(40) and P. aeruginosa (32).

Colistin is used not only in the human field but also in veterinary medicine and its wide use in some countries facilitated the selection of resistant strains and the transmission from animals and environments to humans (29). A Chinese study, investigating the spreading of antimicrobial resistant isolates in a high-density transit area, highlighted that public transportation is a reservoir of $\mathrm{mcr}$ 1 positive bacteria (31). In another study, also carried out in China in 2017, it was found that $34.1 \%$ of Musca domestica and $51.1 \%$ of Protophormia terraenovae homogenates are positive for $\mathrm{mcr}-1$. (45), thus suggesting that these insects could play a role in the dissemination of plasmid in various ecological niches $(16,26)$. It has recently been highlighted that $m c r-1$ is associated with other resistance determinants, e.g. NDM-5 in the United States (25), and that this would make the situation even more critical.

In Italy, the first case of $E$. coli $m c r-1$ positive strain was described in 2016 in a study in which the authors carried out a screening of colistin-resistant isolates showing that eight out of nine $E$. coli strains tested, were $m c r-1$ positive (6). In a study presented at the ECCMID in Vienna 2017, performed by the University of Palermo, the authors demonstrated a prevalence of $4.1 \%$ of $m c r-1$ positive isolates on meat for human consumption (17). In the period August 2016-January 2017, three cases of sepsis by $m c r-1$ positive bacteria have been described in the Pavia hospital (11). It should be underlined that the three patients did not present any risk factor or relationship, thus suggesting that the presence of $m c r-1$ is probably underestimated in Italy.

$\mathrm{CF}$ is an autosomal recessive genetic disorder caused by mutations in the trans-membrane conductance regulator (CFTR) gene. Because of the functionally altered protein, the disease is characterized by the formation of thick and viscous mucus in the respiratory tract with a decreased mucociliary clearance and an environment favourable to bacterial colonization. CF airways host pathogen and non-pathogen bacteria resulting in acute or chronic infections. The vicious circle of infections, inflammatory response, overproduction of cytokines and increased bronchial secretions are at the basis of CF lung damage $(9,14)$.

The spectrum of bacterial pathogens colonizing the respiratory tract of CF patients is quite well known. Staphylococcus aureus and Haemophilus influenzae are frequent during the first decade of life. Enterobacteriaceae like E. coli, and $K$. pneumoniae can chronically colonize children's airway; however, their clinical role in the lung disease has still to be defined. P. aeruginosa is a key pathogen especially in older patients since more than $70 \%$ of $\mathrm{CF}$ patients aged $\geq 18$ years are colonized by this bacterium $(19,20,24,46)$. Chronic infection by $P$. aeruginosa is the main cause of the lung function decline and mortality among CF patients
(37). The high frequency of infectious events, whose resolution requires increasingly intense treatments with antimicrobials, results in the emergence of bacterial strains resistant to most of the antibiotics used in clinical practice.

Colistin is an invaluable antibiotic in CF and it is mostly used against $P$. aeruginosa infections, both intravenously for pulmonary exacerbations and aerosolized for chronic maintenance (34). Therefore, the emergence of colistin resistance mediated by transferable elements, such as $m c r-1$, is particularly alarming in this setting. In 2016, the European Centre for Disease Prevention and Control (ECDC) strongly encouraged to reduce the risk of colonization and infection by mor-1 positive bacteria suggesting the "One Health Approach" (an integrated surveillance among hospitals, communities, veterinary, environment and food control). It also prompted the laboratories to develop methods for the $\mathrm{mcr}-1$ gene detection and monitor possible reservoirs of infection. (See: ECDC rapid risk assessment outlines actions to reduce the spread of the $m c r-1$ gene, 2016).

Since CF lung may be a reservoir of colistin-resistant gramnegative bacteria and $m c r-1$ positive strains, the aim of the present study was to evaluate the rate of colistin resistance and the diffusion of $\mathrm{mcr}-1$ gene in $\mathrm{CF}$ context. To that end, colistin resistance was assessed by molecular testing in Enterobacteriaceae (E. coli, $K$. pneumoniae) and $P$. aeruginosa isolated from the respiratory samples of CF patients followed at a pediatric hospital in the period March 2017- February 2018.

\section{Materials and Methods}

\section{Bacterial isolates}

Colistin-resistant gram-negative bacterial isolates were collected from respiratory samples from CF patients in follow-up at the CF Centre of Children's Hospital Bambino Gesù of Rome that takes care of about 350 patients aged from 0 to 57 years old. Bacterial identification was performed, in accordance with internal procedures, by MALDI-TOF MS (Bruker Daltonics Inc. Billerica, MA USA).

\section{In vitro susceptibility testing}

Susceptibility to colistin was evaluated, in accordance with EUCAST recommendations, by broth dilution method (SensiTest Colistin, Liofilchem, Roseto degli Abruzzi, Italy). Results were interpreted according to the new MIC breakpoints recently proposed by EUCAST: MIC values $\leq 2 \mu \mathrm{g} / \mathrm{mL}$ and $>2 \mu \mathrm{g} / \mathrm{mL}$ categorized the isolates as sensitive or resistant, respectively. An isolate was also classified as (23): i) multidrug-resistant (MDR), if resistant to at least 1 agent in $\geq 3$ antibiotic classes; ii) extensively drugresistant (XDR), if non-susceptible to at least 1 agent in all but $\leq 2$ antibiotic classes; iii) pandrug-resistant (PDR), if resistant to all agents in all antimicrobial classes.

\section{Molecular tests}

DNA was extracted from bacterial colonies using magnetic particles (EZ1 DNA Extraction Mini Kit, and Biorobot EZ-1 workstation, Qiagen, Germany), according to the manufacturer's instructions, and then stored at $-80^{\circ} \mathrm{C}$ until analysis. MICROSEQ $50016 \mathrm{~S}$ rDNA bact. Seq. (Life Technologies, Italy) was used to check the outcome of the DNA extraction. PCR was performed to detect $m c r-1$ gene using primers (forward: 5'CGGTCAGTCCGTTTGTTC'3; reverse: 5'CTTGGTCGGTCTGTAGGG'3) and conditions previously described (22). PCR products were run using Flash gels (Lonza, USA) considering $m c r-1$ positive samples those 
showing a band at 309 bps. P. aeruginosa ATCC27853 and E. coli ATCC13846 were used as negative control, whereas a $m c r-1$ positive E. coli strain, kindly donated by Istituto Zooprofilattico Sperimentale Lazio e Toscana, was used as positive control.

\section{Results}

A total of 47, consecutive, non-duplicated colistin-resistant gram-negative bacterial isolates from respiratory samples of $39 \mathrm{CF}$ patients were screened: $42(89.4 \%)$ P. aeruginosa, $4(8.5 \%) \mathrm{K}$. pneumoniae and one (2.1\%) E. coli.

Results from antibiotic susceptibility testing are summarized in Table 1. Among 47 colistin-resistant isolates, 13 (27.6\%) were classified as MDR (10 P. aeruginosa, $2 \mathrm{~K}$. pneumoniae, one $E$. coli), 9 (19.1\%) as XDR (7 P. aeruginosa; 2 K. pneumoniae), and 9 (19.1\%) were PDR (all P. aeruginosa).

$P$. aeruginosa isolates showed MIC values ranging from 4 to $\geq 16 \mu \mathrm{g} / \mathrm{mL}$; we found MIC values equal to $4 \mu \mathrm{g} / \mathrm{mL}$ in 13 isolates, and MIC values equal to or greater than $8 \mu \mathrm{g} / \mathrm{mL}$ (low- and highlevel colistin resistance, respectively) in 29 isolates. $E$. coli and $K$. pneumoniae isolates showed a colistin MIC equal to $8 \mu \mathrm{g} / \mathrm{mL}$ and $\geq 16 \mu \mathrm{g} / \mathrm{mL}$, respectively.

No correlation was observed between MIC values and resistance profiles (MDR, XDR, PDR) (Table 2).

None of the 47 isolates resulted to be positive for the $m c r-1$ gene (Figure 1).

\section{Discussion}

The antibiotic colistin, rarely used in the past, today represents one of the last resort agents to combat multi- or even extensivelydrug-resistant bacteria, as carbapenem-resistant Enterobacteriaceae, A. baumannii and P. aeruginosa. Unfortunately, colistin-resistant gram-negative bacterial strains have been already detected in several clinical contexts, especially those with a high intensity of care namely Intensive Care and Complex Chronic Diseases Units - complicating the treatment of multiple life-threating conditions.

Colistin is used in CF patients chronically colonized by $P$. aeruginosa both intravenously to treat pulmonary exacerbations and nebulized for maintenance therapies.

In our CF Centre, out of 163 patients colonized by $P$. aeruginosa (Report 2017), 68 (41.7\%), receive colistimethate dry powder for inhalation twice daily. The aerosol administration of antibiotics confers the advantage of reaching high concentrations of the drug in the lung, reducing systemic exposure and toxicity. Among patients treated by inhaled colistin in our CF Centre, 39 subjects $(57.3 \%)$ developed resistance toward this agent. Even if some studies have demonstrated that the risk of resistance occurrences by inhalatory therapies are less common $(10,30)$, we cannot exclude that the high prevalence of colistin-resistant $P$. aeruginosa isolates we observed could be due to the chronic and intensive usage of colistin.

Recently, EUCAST has coined the term "Area of Technical Uncertainty" (ATU) to warn microbiologists about uncertain interpretation of antimicrobial susceptibility testing results. The ATU is defined by one MIC-value in an area where evaluation and reproducibility are difficult to achieve. For $P$. aeruginosa and colistin the ATU is $4 \mu \mathrm{g} / \mathrm{mL}$ (www.eucast.org). Among the bacterial isolates belonging to our collection, 13 P. aeruginosa isolates showed a MIC equal to $4 \mu \mathrm{g} / \mathrm{mL}$. EUCAST didn't set mandatory actions to perform in this scenario. In our practice, we report the results as resistant ("R") and take the opportunity to discuss the case with clinical colleagues during the daily meeting.

It should be noted that MIC reflects a serum achievable measurement of resistance that may not be applicable to the inhaled route of administration. Pharmacokinetics studies have shown high concentrations of colistin in the lung of mechanically ventilated critically ill patients undergoing inhaled colistin therapy (2). Particularly, colistin concentrations in the epithelial lining fluid were always higher than the MIC value and five-fold higher than those in serum. Therefore, we are reassured that, especially for the maintenance therapies, a MIC value of $4 \mu \mathrm{g} / \mathrm{mL}$ should not be a big concern in our context. In fact, the colistin concentration in the airways during inhaled therapies should be higher than $4 \mu \mathrm{g} / \mathrm{mL}$ leading to the consideration that ATU values should not have a strong impact in clinical outcomes. Anyway, we still maintain close communication with the clinicians in these cases.

Finally, the evidence that, even presenting a high percentage of MDR, XDR or PDR strains, none of our patients has not yet been affected by a gram-negative isolate carrier of $m c r-1$ gene confirms
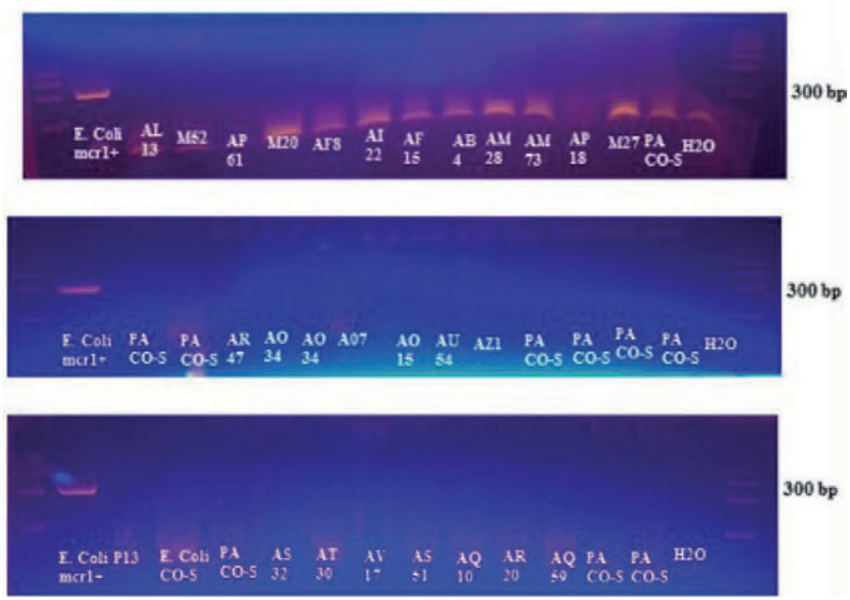

Figure 1. PCR amplification products for mcr-1 gene of colistin resistant Gram-negatives strains isolated from respiratory secretions of CF patients. PA CO-S=colistin-susceptible Pseudomonas aeruginosa; E. coli CO-S=colistin-susceptible Escherichia coli. 
Table 1. Phenotypic and genotypic characteristics of the bacterial isolates studied.

\begin{tabular}{|c|c|c|c|c|c|c|}
\hline Isolates ID & $\begin{array}{l}\text { Bacterial } \\
\text { species }\end{array}$ & Sample & $\begin{array}{l}\text { Patient age } \\
\text { (yrs) }\end{array}$ & $\begin{array}{l}\text { Colony and resistance } \\
\text { phenotypes }\end{array}$ & $\begin{array}{l}\text { Colistin MIC } \\
(\mu \mathrm{g} / \mathrm{mL})\end{array}$ & $\begin{array}{l}\text { Resistance } \\
\text { phenotype }\end{array}$ \\
\hline AZ1 & P. aeruginosa & Tracheal aspirate & 8 & Small Colony Variant & 4 & Carbapenem \\
\hline AL13 & P. aeruginosa & Tracheal aspirate & 14 & Round & 4 & Fluoroquinolone; Aminoglycoside \\
\hline M25 & P. aeruginosa & Sputum & 16 & Mucoid & 4 & Carbapenem \\
\hline $\mathrm{AB} 15$ & P. aeruginosa & Tracheal aspirate & 19 & & 4 & PDR \\
\hline AG18 & P. aeruginosa & Tracheal aspirate & 17 & & 4 & Carbapenem \\
\hline M20 & P. aeruginosa & Tracheal aspirate & 7 & & 4 & \\
\hline AP61 & P. aeruginosa & Tracheal aspirate & 13 & & 4 & \\
\hline AN11 & P. aeruginosa & Tracheal aspirate & 1 & & 4 & Carbapenem \\
\hline AN43 & P. aeruginosa & Sputum & 12 & & 4 & \\
\hline AL56 & P. aeruginosa & Sputum & 20 & & 4 & PDR \\
\hline AP18 & P. aeruginosa & Sputum & 17 & & 4 & Carbapenem \\
\hline AB4 & P. aeruginosa & Sputum & 29 & Small Colony Variant & 4 & Fluoroquinolone; Aminoglycoside \\
\hline M52 & P. aeruginosa & Sputum & 28 & & 4 & PDR \\
\hline AM73 & P. aeruginosa & Sputum & 27 & & 8 & MDR \\
\hline AM54 & P. aeruginosa & Sputum & 15 & & 8 & PDR \\
\hline AM28 & P. aeruginosa & Sputum & 31 & Fringed & 8 & Carbapenem \\
\hline $\mathrm{AO} 15$ & P. aeruginosa & Tracheal aspirate & 15 & Small Colony Variant & $>8$ & PDR \\
\hline AB43 & P. aeruginosa & Sputum & 12 & & $>8$ & Carbapenem \\
\hline AN35 & P. aeruginosa & Sputum & 27 & Small Colony Variant & $>8$ & Fluoroquinolone \\
\hline M27 & P. aeruginosa & Sputum & 30 & Fringed & $>8$ & MDR \\
\hline AN14 & P. aeruginosa & Sputum & 25 & Smooth & $>8$ & PDR \\
\hline AF8 & P. aeruginosa & Sputum & 26 & Round & $>8$ & XDR \\
\hline$\overline{A B} 26$ & P. aeruginosa & Sputum & 29 & 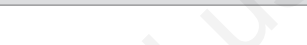 & $>8$ & XDR \\
\hline AN12 & $P$. aeruginosa & Sputum & 12 & Smooth & $>8$ & MDR \\
\hline $\mathrm{AI} 22$ & P. aeruginosa & Sputum & 16 & Small Colony Variant & $>8$ & \\
\hline AF15 & P. aeruginosa & Sputum & 39 & 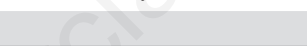 & $>8$ & MDR \\
\hline AF5 & P. aeruginosa & Sputum & 34 & 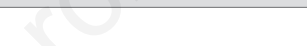 & $>8$ & \\
\hline AE26 & $P$. aeruginosa & Sputum & 37 & Small Colony Variant & $>8$ & XDR \\
\hline A018 & P. aeruginosa & Sputum & 28 & Mucoid & $\geq 8$ & MDR \\
\hline AQ59 & P. aeruginosa & Sputum & 29 & & $\geq 16$ & PDR \\
\hline AR47 & P. aeruginosa & Sputum & 20 & Round & $\geq 16$ & MDR \\
\hline $\mathrm{AO} 34$ & P. aeruginosa & Sputum & 15 & & $\geq 16$ & PDR \\
\hline $\mathrm{AO7}$ & P. aeruginosa & Sputum & 13 & & $\geq 16$ & MDR \\
\hline AS51 & P. aeruginosa & Sputum & 16 & & $\geq 16$ & PDR \\
\hline AQ10 & P. aeruginosa & Sputum & 32 & & $\geq 16$ & XDR \\
\hline ALM01 & P. aeruginosa & Sputum & 35 & & $\geq 16$ & MDR \\
\hline AR20 & P. aeruginosa & Sputum & 28 & & $\geq 16$ & MDR \\
\hline AS45 & $P$. aeruginosa & Sputum & 27 & & $\geq 16$ & XDR \\
\hline AU54 & P. aeruginosa & Sputum & 21 & Small Colony Variant & $\geq 16$ & XDR \\
\hline AT30 & P. aeruginosa & Sputum & 16 & Small Colony Variant & $\geq 16$ & XDR \\
\hline AV17 & P. aeruginosa & Sputum & 14 & Small Colony Variant & $\geq 16$ & MDR \\
\hline AS32 & P. aeruginosa & Sputum & 16 & Mucoid & $\geq 16$ & Carbapenem \\
\hline $\mathrm{P} 13$ & E. coli & Tracheal aspirate & 16 & ESBL+ & 8 & XDR \\
\hline P61 & K. pneumoniae & Tracheal aspirate & 1 & ESBL+ & $\geq 16$ & MDR \\
\hline S19 & K. pneumoniae & Tracheal aspirate & 1 & ESBL- HODGE- MBL+ & $\geq 16$ & XDR \\
\hline R31 & K. pneumoniae & Tracheal aspirate & 1 & ESBL+ & $\geq 16$ & XDR \\
\hline P63 & K. pneumoniae & Pharyngeal swab & 1 & $\mathrm{KPC}+$ & $\geq 16$ & MDR \\
\hline
\end{tabular}

MIC, Minimum inhibitory concentration.

Table 2. Minimum inhibitory concentration (MIC) value and category of resistance for $P$ aeruginosa isolates.

\begin{tabular}{|c|c|c|c|}
\hline MIC & MDR & XDR & PDR \\
\hline $4 \mu \mathrm{g} / \mathrm{mL}$ & 0 & 0 & 3 \\
\hline$\geq 8 \mu \mathrm{g} / \mathrm{mL}$ & 5 & 3 & 3 \\
\hline$\geq 16 \mu \mathrm{g} / \mathrm{mL}$ & 5 & 4 & 3 \\
\hline
\end{tabular}


the effectiveness of prevention and control measures for crossinfection implemented in our CF Centre. The segregation policy adopted by most of CF centres, as already known, is a winning strategy for reducing the incidence of hospital-acquired infections.

Subsequently to the first report on $m c r-1$ gene in 2015 in China, in July 2016 a new plasmid-mediated gene was identified in Belgium in a colistin-resistant $E$. coli strain porcine that was negative for $m c r-1$. The gene, named $m c r-2$, is a phosphoethanolaminetransferase found in the IncX4 plasmid, which seems to have a common acquisition pattern with the $m c r-1$. It shows $76.7 \%$ homology with $m c r-1$ and in Belgium its prevalence in colistin-resistant $E$. coli resulted to be higher than that of $m c r-1$ (41).

After the identification of further determinants of resistance to colistin, named $m c r-3$ and $m c r-4$, in September 2017 a German study identified an additional phosphoethanolamine-transferase associated transposone, named $m c r-5$, which confers resistance to colistin in Salmonella paratyphi B d Ta+ strains $(4,7,43)$. Additionally, $m c r-6 / 7 / 8$ have been recently identified $(1,38,42)$.

\section{Conclusions}

In the light of these new reports, although our results provided evidence about $m c r-1$ gene absence we cannot have information about other genetic determinants $m c r-2 / 3 / 4 / 5 / 6 / 7 / 8$ (27).

In conclusion, if from one hand results arose from this survey reassure us, on the other they strengthen the need to follow the same path for the other determinants of resistance.

\section{References}

1. Abu Oun M, Stubberfield EJ, Duggett NA, et al. mcr-1 and mcr-2 (mcr-6.1) variant genes identified in Moraxella species isolated from pigs in Great Britain from 2014 to 2015. J Antimicrob Chemother 2018;73:2904.

2. Athanassa ZE, Markantonis SL, Tsakri A. Pharmacokinetics of inhaled colistimethate sodium (CMS) in mechanically ventilated critically ill patients. Intensive Care Med 2012;38:1779-86.

3. Beceiro A, Moreno A, Fernández N, et al. Biological cost of different mechanisms of colistin resistance and their impact on virulence in Acinetobacter baumannii. Antimicrob Agents Chemother 2014;58:518-26.

4. Borowiak M, Fischer J, Hammerl JA, et al. Identification of a novel transposon-associated phosphoethanolamine transferase gene, mcr-5, conferring colistin resistance in d-tartrate fermenting Salmonella enterica subsp. enterica serovar Paratyphi B. J Antimicrob Chemother 2017;72:3317-24.

5. Caniaux I, van Belkum A, Zambardi G, et al. MCR: modern colistin resistance. Eur J Clin Microbiol Infect Dis 2017;36:415-20.

6. Cannatelli A, Giani T, Antonelli A, et al. First detection of the mcr-1 colistin resistance gene in Escherichia coli in Italy. Antimicrob Agents Chemother 2016;60:3257-8.

7. Carretto E, Brovarone F, Nardini P, et al. Detection of mcr-4 positive Salmonella enterica serovar Typhimurium in clinical isolates of human origin, Italy, October to November 2016. Euro Surveill 2018;23.

8. Castanheira M, Griffin MA, Deshpande LM, et al. Detection of mcr-1 among Escherichia coli Clinical Isolates Collected Worldwide as Part of the SENTRY Antimicrobial Surveillance Program in 2014 and 2015. Antimicrob Agents Chemother 2016;60:5623-4.
9. Castellani C, Assael BM. Cystic fibrosis: a clinical view. Cell Mol Life Sci 2017;74:129-40.

10. Conole D, Keating GM. Colistimethate sodium dry powder for inhalation: A review of its use in the treatment of chronic pseudomonas aeruginosa infection in patients with cystic fibrosis. Drugs 2014;74:377-87.

11. Corbella M, Mariani B, Ferrari C, et al. Three cases of mcr-1positive colistin-resistant Escherichia coli bloodstream infections in Italy, August 2016 to January 2017. Euro Surveill 2017;22:30517.

12. Eiamphungporn W, Yainoy S, Jumderm C, et al. Prevalence of the colistin resistance gene mcr-1 in colistin-resistant Escherichia coli and Klebsiella pneumoniae isolated from humans in Thailand. J Glob Antimicrob Resist 2018;15:32-5.

13. El Garch F, Sauget M, Hocquet D, et al. mcr-1 is borne by highly diverse Escherichia coli isolates since 2004 in food-producing animals in Europe. Clin Microbiol Infect 2017;23:51.

14. Elborn JS. Cystic fibrosis. Lancet 2016;388:2519-31.

15. Falagas ME, Kasiakou SK. Toxicity of polymyxins: a systematic review of the evidence from old and recent studies. Crit Care 2006;10:R27.

16. Fang Y, Zhang Y, Zhou ZB, et al. New strains of Japanese encephalitis virus circulating in Shangai, China after a ten-year hiatus in local mosquitos surveillance. Parasit Vectors 2019; $12: 22$.

17. Ghodousi A, Bonura C, Mammina C. Presence of mcr-1-positive Escherichia coli in retail meat in Italy. 10.13140/ RG.2.2.12767.87206. Vienna, Austria, ECCMID 2017.

18. Gupta S, Govil D, Kakar PN, et al. Colistin and polymyxin B: A re-emergence. Indian J Crit Care Med 2009;13:49-53.

19. Harrison F. Microbial ecology of the cystic fibrosis lung. Microbiology 2007;153:917-23.

20. Hoo ZH, Edenborough FP, Curley R, et al. Understanding Pseudomonas status among adults with cystic fibrosis: a realworld comparison of the Leeds criteria against clinicians' decision. Eur J Clin Microbiol Infect Dis 2018;37:735-43.

21. Jansen G, Mahrt N, Tueffers L, et al. Association between clinical antibiotic resistance and susceptibility of Pseudomonas in the cystic fibrosis lung. Evol Med Public Health 2016:182-94.

22. Le J, Turner N, Deshpande LM, et al. Case report of transient mcr-1-haboring Escherichia coli with concurrent Staphylococcus aureus bacteremia in Long Beach, California. Diagn Microbiol Infect Dis 2017;89:303-4.

23. Liu YY, Wang Y, Walsh TR, et al. Emergence of plasmid-mediated colistin resistance mechanism MCR-1 in animals and human beings in China: a microbiological and molecular biological study. Lancet Infect Dis 2016;16:161-8.

24. Magiorakos AP, Srinivasan A, Carey RB et al. Multidrug-resistant, extensively drug-resistant and pandrug-resistant bacteria: an international expert proposal for interim standard definitions for acquired resistance. Clin Microbiol Infect 2012;18: 268-81.

25. Mahenthiralingam E. Emerging cystic fibrosis pathogens and the microbiome. Paediatr Respir Rev 2014;15:13-5.

26. Mediavilla JR, Patrawalla A, Chen L, et al. Colistin- and carbapenem-resistant Escherichia coli harboring mor-1 and blaNDM-5, causing a complicated urinary tract infection in a patient from the United States. MBio 2016;7:e01191-216.

27. Mohlmann TWR, Oymans J, Wichgers Schreur PJ, et al. Vector competence of biting midges and mosquitoes for Shuni virus. PloS Negl Trop Dis 2019;13:e006609.

28. Pedersen MG, Olesen HV, Jensen-Fangel S, et al. Colistin resistance in Pseudomonas aeruginosa and Achromobacter spp. cultured from Danish cystic fibrosis patients is not related 
to plasmid-mediated expression of mcr-1. J Cyst Fibros 2018; 17:e22-3.

29. Olaitan AO, Morand S, Rolain JM. Mechanisms of polymyxin resistance: acquired and intrinsic resistance in bacteria. Front Microbiol 2014;5:643.

30. Poirel L, Jayol A, Nordmann P. Polymyxins: antibacterial activity, susceptibility testing, and resistance mechanisms encoded by plasmids or chromosomes. Clin Microbiol Rev 2017;30:557-96.

31. Schuster A, Haliburn C, Döring G et al. Safety, efficacy and convenience of colistimethate sodium dry powder for inhalation (colobreathe DPI) in patients with cystic fibrosis: A randomised study. Thorax 2013;68:344.

32. Shen C, Feng S, Chen H, et al. Transmission of mcr-1-Producing Multidrug-resistant Enterobacteriaceae in Public Transportation in Guangzhou, China. Clin Infect Dis 2018;67:S217-24.

33. Snesrud E, Maybank R, Kwak YI, et al. Chromosomally encoded mcr-5 in colistin-nonsusceptible Pseudomonas aeruginosa. Antimicrob Agents Chemother 2018;62:8.

34. Srijan A, Margulieux KR, Ruekit S, et al. Genomic Characterization of Nonclonal mcr-1-Positive MultidrugResistant Klebsiella pneumoniae from Clinical Samples in Thailand. Microb Drug Resist 2018;24:403-10.

35. Taccetti G, Campana S, Neri AS, et al. Antibiotic therapy against Pseudomonas aeruginosa in cystic fibrosis. J Chemother 2008;20:166-9.

36. Tada T, Nhung PH, Shimada K, et al. Emergence of colistinresistant Escherichia coli clinical isolates harboring mcr-1 in Vietnam. Int J Infect Dis 2017;63:72-3.

37. Tada T, Uechi K, Nakasone I, et al. Emergence of a colistin- resistant Escherichia coli clinical isolate harboring mcr-1 in Japan. Int J Infect Dis 2017;63:21-2.

38. Ullrich G, Wiedau-Görs S, Steinkamp G, et al. Parental fears of Pseudomonas infection and measures to prevent its acquisition. J Cyst Fibros 2002;1:122-30.

39. Wang X, Wang Y, Zhou Y, et al. Emergence of a novel mobile colistin resistance gene, mcr-8, in NDM-producing Klebsiella pneumoniae. Emerg Microbes Infect 2018;7:122.

40. Wong SC, Tse H, Chen JH, et al. Colistin-resistant Enterobacteriaceae carrying the mcr-1 gene among patients in Hong Kong. Emerg Infect Dis 2016;22:1667-9.

41. Xavier BB, Lammens C, Ruhal R, et al. Identification of a novel plasmid-mediated colistin-resistance gene, $\mathrm{mcr}-2$, in Escherichia coli, Belgium, June 2016.Euro Surveill 2016;21.

42. Yang YQ, Li YX, Lei CW, et al. Novel plasmid-mediated colistin resistance gene mcr-7.1 in Klebsiella pneumoniae. J Antimicrob Chemother 2018;73:1791-5.

43. Yin W, Li H, Shen Y, et al. Novel Plasmid-Mediated Colistin Resistance Gene mcr-3 in Escherichia coli. MBio 2017;8: e00543-17.

44. Yu CY, Ang, GY, Chin PS, et al. Emergence of mcr-1-mediated colistin resistance in Escherichia coli in Malaysia. Int $\mathrm{J}$ Antimicrob Agents 2016;47:504-5.

45. Zhang J, Wang J, Chen L, et al. Housefly (Musca domestica) and Blow Fly (Protophormia terraenovae) as Vectors of Bacteria Carrying Colistin Resistance Genes. Appl Environ Microbiol 2017;84:e01736-17.

46. Zhao J, Schloss PD, Kalikin LM, et al. Decade-long bacterial community dynamics in cystic fibrosis airways. Proc Natl Acad Sci USA 2012;109:5809-14. 